\title{
An astronomical search for evidence of new physics: Limits on gravity-induced birefringence from the magnetic white dwarf RE J0317-853
}

\author{
Oliver Preuss, ${ }^{1}$ Mark P. Haugan, ${ }^{2}$ Sami K. Solanki, ${ }^{1}$ and Stefan Jordan ${ }^{3,4}$ \\ ${ }^{1}$ Max-Planck-Institut für Aeronomie, D-37191 Katlenburg-Lindau, Germany \\ ${ }^{2}$ Department of Physics, Purdue University 1396, West Lafayette, Indiana 47907, USA \\ ${ }^{3}$ Universität Tübingen, Institut für Astronomie und Astrophysik, D-72076 Tübingen, Germany \\ ${ }^{4}$ Astronomisches Rechen-Institut, D-69120 Heidelberg, Germany
}

(Dated: October 29, 2018)

\begin{abstract}
The coupling of the electromagnetic field directly with gravitational gauge fields leads to new physical effects that can be tested using astronomical data. Here we consider a particular case for closer scrutiny, a specific nonminimal coupling of torsion to electromagnetism, which enters into a metric-affine geometry of space-time. We show that under the assumption of this nonminimal coupling, spacetime is birefringent in the presence of such a gravitational field. This leads to the depolarization of light emitted from extended astrophysical sources. We use polarimetric data of the magnetic white dwarf RE J0317-853 to set for the very first time constraints on the essential coupling constant for this effect, giving $k^{2} \lesssim(19 \mathrm{~m})^{2}$.
\end{abstract}

PACS numbers: 04.80.Cc

\section{INTRODUCTION}

The designation new physics is used here to refer to phenomena beyond the scope of the standard model of particle physics or of general relativity. Today, efforts to develop a quantum theory of gravity or a complete, consistent and unified theory of matter and all its interactions are rich and compelling sources of speculation about new physics. Certainly, the effective field theories that emerge as low-energy limits of string theories are littered with new fields and interactions. Since cherished symmetries like CPT and Lorentz invariance can be broken in these contexts [1, 3], high-precision experimental and observational tests of these symmetries offer particularly effective ways of searching for evidence of new physics.

In this letter we focus on consequences of interactions that couple the electromagnetic field to new physics. In recent papers by Kostelecky \& Mewes 2], couplings of the electromagnetic field to an axial-vector, $\left(k_{A F}\right)^{\mu}$, and to a tensor, $\left(k_{F}\right)^{\mu \nu \sigma \tau}$, are used to provide a generic characterization of conceivable new physics influencing the photon sector. The $k_{A F}$ and $k_{F}$ structures could, for example, be constructs of vector and tensor fields in an underlying theory. If such fields acquire nonzero vacuum expectation values, their couplings to the electromagnetic field spontaneously break global Lorentz invariance. The $k_{A F}$ structure breaks CPT as well. While motivated by current speculations regarding fundamental physics, the conceptual framework and formalism of the work by Kostelecky and Mewes is that of Lagrangian-based local field theory. With different motivations, questions regarding the most general way in which new fields may couple to the electromagnetic field have been addressed in this framework before. It is reassuring to find all of these studies reaching equivalent conclusions.

Today's strong empirical foundation for general relativity is a consequence of continuing efforts to probe for evidence of this sort of new or alternative physics, see Will [4] for a review. In this research setting the Lagrangian-based local field theory framework is often called the Dicke framework [4]. Theoretical alternatives to general relativity can feature gravitational fields in addition to the symmetric second-rank tensor potential of general relativity. One distinguishes two classes of alternative theories based on the way in which these gravitational fields couple to matter. A theory is metric if it admits a representation in which matter fields are all minimally coupled in the familiar way to a symmetric secondrank tensor gravitational field alone, implying that auxiliary fields couple only to that metric tensor field and to each other. Otherwise, a theory is nonmetric. It is in the study of nonmetric theories which couple gravitational fields directly to the electromagnetic field that one finds analogues of the recent work by Kostelecky and Mewes. The equivalent of their Lagrangian density governing the dynamics of the electromagnetic field can be found in Ni's $\chi g$ formalism [5]. There, this Lagrangian density is expressed in terms of a fourth-rank tensor field, $\chi^{\alpha \beta \gamma \delta}$, constructed from a symmetric second-rank tensor field, $g^{\mu \nu}$, and auxiliary gravitational fields,

$$
\mathcal{L}_{E M}=\chi^{\alpha \beta \gamma \delta} F_{\alpha \beta} F_{\gamma \delta} .
$$

$\mathrm{Ni}$ [6] noted that theories encompassed by this formalism can predict birefringence and used pulsar polarization observations to constrain this possibility twenty years ago.

Recognition that gravitation theories touted as viable alternatives to general relativity could predict such birefringence stimulated new interest in such possibilities in the 1990s and sharp constraints were imposed on birefringence predicted by Moffat's non-symmetric gravitation theory (NGT) 7, [8, 9, 10, 11]. The analysis of birefringence by Kostelecky and Mewes [2] and that performed by Haugan and Kauffmann [11] in the general context provided by the $\chi g$ formalism run parallel to one another. Indeed, the $3+1$ decomposition of the $\chi$ tensor made by 
Haugan and Kauffmann yields $\mathrm{SO}(3)$ tensors equivalent to the $\kappa$ matrices of Kostelecky and Mewes,

$$
\left(\kappa_{D E}\right)^{i j}=2 \xi^{i j},\left(\kappa_{H B}\right)^{i j}=2 \zeta^{i j},\left(\kappa_{D B}\right)^{i j}=2 \gamma^{i j} .
$$

Kostelecky and Mewes restrict their attention to cases in which these quantities are spacetime constants. Haugan and Kauffmann allow them to vary, reflecting the structure of fields involved in new physics that are generated by localized sources. Their work establishes that evidence of such birefringence can reveal new physics even when the fields involved do not acquire nonzero vacuum expectation values and global Lorentz invariance is not spontaneously broken. String-inspired speculation regarding new physics provides a compelling motivation to continue searching for evidence of birefringence of the kind analyzed in preceding references.

As additional motivation we offer a new example, drawn from the gravitation physics literature, of a theoretical alternative to general relativity that naturally predicts such birefringence if we assume a special nonminimal coupling of torsion to electromagnetism. Besides Moffat's NGT this provides a new concrete test case against which one can gauge the precision of birefringence searches. In this context it is important to note that the new version of Moffat's NGT [12] also provides a valid alternative to general relativity which has overcome the technical difficulties found by Damour et al. 13] and Clayton et al. [14]. The foil for general relativity we present in section II, is drawn from the class of metricaffine theories of gravity [15]. We hope that this example will prompt further work on gravity-induced birefringence predicted by metric-affine theories and other alternatives to general relativity; see [16]. In this paper we also report new observational constraints on such gravityinduced birefringence based on data from white dwarfs which constitute the most abundant stage of final stellar evolution - possessing a strong magnetic field.

\section{GRAVITY-INDUCED BIREFRINGENCE IN METRIC-AFFINE THEORIES}

Members of the class of metric-affine theories of gravity feature torsion and/or nonmetricity gravitational fields in addition to a symmetric second-rank tensor gravitational potential [15. The minimal or geometrical coupling scheme for coupling gravity to matter in metric-affine theories does couple torsion and nonmetricity to some matter fields so metric-affine theories are, in fact, nonmetric theories. However, the minimal coupling scheme does not couple torsion or nonmetricity to the electromagnetic field [18]. Only nonminimally coupled metricaffine theories can conceivably predict birefringence.

Here, we focus entirely on the effect of nonminimal coupling to torsion. We suggest, in addition to the conventional Maxwell Lagrangian, the additional nonminimal piece [26]

$$
L_{E M}=k^{2} *\left(T_{\alpha} \wedge F\right) T^{\alpha} \wedge F
$$

see [17] for other possibilities, where $k$ is a coupling constant with the dimension of length, $*$ denotes the Hodge dual, $T$ denotes the torsion and $F$ the electromagnetic field, which is related in the usual way to its potential $A$. This addition is consistent with gauge invariance and, so, with charge conservation. It corresponds to a term

$$
\delta \mathcal{L}_{E M}=\delta \chi^{\alpha \beta \gamma \delta} F_{\alpha \beta} F_{\gamma \delta},
$$

in the notation of Haugan and Kauffmann [1]. This correspondence permits us to infer consequences of the nonminimal torsion coupling above from the results of the general analysis of reference 11]. Since our immediate interest is the interpretation of stellar data, we focus on the case of static, spherically symmetric metric-affine fields to explore the dominant effects of solar torsion on the propagation of light from the Sun. Tresguerres 19] has expressed the most general form of a static, spherically symmetric torsion field in terms of six radial functions. In his notation these are $\alpha, \beta$ and $\gamma_{(i)}$ for $i=1$ to 4. Plugging his representation into $\delta \mathcal{L}_{E M}$ above yields

$$
\begin{aligned}
\delta \mathcal{L}_{E M} & =k^{2}\left\{\left(\alpha^{2}-\beta^{2}\right) B_{1}^{2}-\left(\gamma_{(1)}^{2}+\gamma_{(4)}^{2}\right)\left[B_{2}^{2}+B_{3}^{2}\right]\right. \\
& -\left(\gamma_{(3)}^{2}+\gamma_{(4)}^{2}\right)\left[E_{2}^{2}+E_{3}^{2}\right] \\
& +2\left(\gamma_{(1)} \gamma_{(4)}-\gamma_{(2)} \gamma_{(3)}\right)\left[B_{2} E_{2}+B_{3} E_{3}\right] \\
& \left.+2\left(\gamma_{(1)} \gamma_{(3)}+\gamma_{(2)} \gamma_{(4)}\right)\left[B_{3} E_{2}-B_{2} E_{3}\right]\right\}
\end{aligned}
$$

where $E$ and $B$ refer in the usual way to the electric and magnetic components of $F$ in Tresguerres' coordinates [26, 27]. Note that the three-vector index 1 refers to the radial direction. In terms of the components of the $\xi$, $\zeta$ and $\gamma$ tensors of reference [11], we can read off from the corresponding expression for $\delta \mathcal{L}_{E M}$ how the nonzero components of the tensors $\xi, \zeta$ and $\gamma$ are expressed in terms of the Tresguerres functions for the nonminimal coupling considered here. To make a specific prediction of the effect this torsion coupling will have on the propagation of light from a star, we must consider a specific metric-affine candidate for the stellar field. We choose a special case of a static, spherically symmetric field found by Tresguerres [20] in which nonmetricity vanishes, corresponding to vanishing dilatation and shear charge. For simplicity we also set the cosmological constant to zero. The corresponding forms of Tresguerres' $\alpha, \beta$ and $\gamma_{(i)}$ functions are

$$
\begin{aligned}
\alpha(r) & =\frac{1}{r}-\frac{m}{r^{2}}, \quad \beta(r)=\frac{1}{r}+\frac{m}{r^{2}} \\
\gamma_{(1)}(r) & =-\frac{1}{2}\left(\frac{1}{r}+\frac{m}{r^{2}}\right), \gamma_{(3)}(r)=\frac{1}{2}\left(\frac{1}{r}-\frac{m}{r^{2}}\right)
\end{aligned}
$$

with $\gamma_{(2)}=\gamma_{(4)}=0$. A somewhat tedious recapitulation of the analysis in 11] in this case leads to the conclusion that, to leading order, the specified nonminimal torsion coupling and chosen stellar torsion field singles out linear polarizations with a fractional difference in their propagation speeds of

$$
\frac{\delta c}{c}=-\sqrt{6} \frac{k^{2} m}{r^{3}} \sin ^{2}(\theta),
$$


where $\theta$ denotes the angle relative the outward radial direction in which the light is propagating. In this context it is interesting to compare (8) with equation (21) and (22) of [16].

We are interested in the effect that this differential propagation has on the polarization of light as it travels from a localized source on the stellar surface to an observer. This is determined by the phase shift that accumulates between the polarization components singled out by the stellar field as the light propagates. Recapitulating the analysis performed in [9, 10], we find that in this case

$$
\Delta \Phi=\sqrt{\frac{2}{3}} \frac{2 \pi k^{2} m}{\lambda R^{2}} \frac{(\mu+2)(\mu-1)}{\mu+1},
$$

where $\mu$ denotes the cosine of the angle between the line of sight and the normal on the stellar surface ( $\mu=1$ : stellar disk center, $\mu=0$ : limb), $\lambda$ is the light's wavelength and $R$ is the stellar radius.

\section{DATA, ANALYSIS AND RESULTS}

All light received from a pointlike source of polarized radiation in a gravitationally birefringent environment suffers the same phase shift $\Delta \Phi(\mu)$. Introducing wavelength dependent Stokes parameters $I_{\lambda}, Q_{\lambda}, U_{\lambda}, V_{\lambda}$ to describe polarized light 21], with Stokes $Q$ defined to represent the difference between linear polarization parallel and perpendicular to the local stellar limb, gravitational birefringence introduces a crosstalk between the linear polarization parameter Stokes $U$ and the net circular polarization, $V$. This crosstalk is such that although the observed values $U_{\text {obs }}$ and $V_{\text {obs }}$ differ from the values emitted by the source, $U_{\text {src }}$ and $V_{\text {src }}$, the composite degree of polarization remains equal: $\left(U_{\mathrm{obs}}^{2}+V_{\mathrm{obs}}^{2}\right)^{1 / 2}=$ $\left(U_{\mathrm{src}}^{2}+V_{\mathrm{src}}^{2}\right)^{1 / 2}$. If an extended source covering a range of $\mu$ values is observed then light emitted from different points suffers different phase shifts and, so, adds up to an incoherent superposition. Summing over the different contributions, while using the additive properties of Stokes parameters, yields a reduction of the observed polarization relative to the light emitted from the source: $\left(U_{\text {obs }}^{2}+V_{\text {obs }}^{2}\right)^{1 / 2}<\left(U_{\mathrm{src}}^{2}+V_{\mathrm{src}}^{2}\right)^{1 / 2}$. Since the rotationally modulated polarization from magnetic white dwarfs can only be produced by an extended source 22] any observed (i.e. non-zero) degree of polarization provides a limit on the strength of birefringence induced by the star's gravitational field [8].

The polarized radiation from white dwarfs is produced at the stellar surface as a result of the presence of ultrastrong (up to $10^{5} \mathrm{~T}$ ) magnetic fields [23]. Since the disk of a white dwarf is unresolved, only the total polarization from all surface elements is observable:

$$
V_{\lambda, \text { tot }}\left(k^{2}\right)=2 \pi \iint V_{\lambda}\left(\mu, B, B_{\|}\right) \cos (\Delta \Phi) \mu d \theta d \phi,
$$

where the Stokes parameter $V_{\lambda}$ changes over the visible hemisphere and depends on the wavelength $\lambda$, the location $\mu$ (limb darkening), the total magnetic field strength $B(\theta, \phi)$, the line-of-sight component $B_{\|}(\theta, \phi)$, and on the parameters of the stellar atmosphere. Gravitational birefringence reduces the polarization by means of $\cos (\Delta \Phi)$. Theoretically, the Stokes parameters can be calculated by solving the radiative transfer equations through a magnetized stellar atmosphere on a large number of surface elements on the visible hemisphere (e.g. 28]). If the star is rotating, the spectrum and polarization pattern changes according to the respective magnetic field distribution visible at a particular moment. To obtain the degree of circular polarization, Eq. (10) has to be divided by the total stellar flux $I_{\lambda, \text { tot }}$ emitted to the observer at wavelength $\lambda$. Below we will calculate a maximum circular polarization $V_{\lambda, \max } / I_{\lambda, \text { tot }}$ from radiative transfer calculations which is in general higher than the observed value $V_{\lambda, \text { obs }} / I_{\lambda, \text { obs }}$. Then we assume that the reduction from $V_{\lambda, \max }$ to $V_{\lambda, \text { obs }}$ is entirely due to the factor $\cos \left(\Delta \Phi\left(k^{2}\right)\right)$ in Eq. (10) thereby calculating the maximum value for $k^{2}$, i.e. our limit on $k^{2}$ is reached as soon as $V_{\lambda \text {,tot }} / I_{\lambda \text {,tot }}$ in Eq. (10) becomes smaller than $V_{\lambda, \text { obs }} / I_{\lambda, \text { tot }}$ for a certain value of $k^{2}$.

RE J0317-853 is a remarkable object within the class of isolated magnetic white dwarfs. Besides being the most rapidly rotating star $(P=725 \mathrm{sec})$ of this type it is also the most massive at $1.35 M_{\odot}$, close to the Chandrasekhar limit [29]. The corresponding radius is only $0.0035 R_{\odot}$. With a reported $V_{\lambda, \text { obs }} / I_{\lambda, \text { tot }}$ of $20 \%$ at $\lambda=576 \mathrm{~nm}$ 31], RE J0317-853 is also the magnetic white dwarf with the highest known level of circular polarization. Due to its small radius and high degree of circular polarization, RE J0317-853 is a very suitable object for setting limits on gravitational birefringence. The analysis of time resolved UV flux spectra obtained with the Hubble Space telescope has shown that the distribution of the field moduli is approximately that of an off-centered magnetic dipole oriented obliquely to the rotation axis with a polar field strength of $B_{d}=3.63 \cdot 10^{4} \mathrm{~T}$, leading to visible surface field strengths between $1.4 \cdot 10^{4} \mathrm{~T}$ and $7.3 \cdot 10^{4} \mathrm{~T}[30$ ]. This model is not only able to describe the UV, but also the optical spectra (Jordan et al. in prep.), which means that the distribution of the magnetic field moduli - but not necessaryly of the longitudinal components - is correctly described. This result is completely independent of the magnitude of the gravitational birefringence. From radiative transfer calculations it follows that at the phase of rotation when the maximum value of $20 \%$ polarization at $576 \mathrm{~nm}$ is measured, almost the entire visible stellar surface is covered by magnetic fields between $1.4 \cdot 10^{4}$ and $2.0 \cdot 10^{4} \mathrm{~T}$, with only a small tail extending to maximum field strengths of $5.3 \cdot 10^{4}$. This distribution can best be modeled by assuming a rotational phase where the axis of the off-centered dipole is nearly perpendicular to the line of sight. Using this special field geometry we calculated a histogram distribution of the visible surface magnetic field strengths in order to set sharp limits on gravita- 
tional birefringence. For each field strength bin of the histogram we calculated the maximum circular polarization from radiative transfer calculations by assuming that the field vector always points towards the observer. The total maximum polarization from the whole visible stellar disk without gravitational birefringence is then calculated by adding up the contributions from each field strength bin weighted with its relative frequency. This results in $V_{\lambda, \max } / I_{\lambda \text {,tot }}=26.5 \%$. Assuming that the reduction to $V_{\lambda, \text { obs }} / I_{\lambda \text {,tot }}=20 \%$ is entirely due to gravity induced depolarization - and not due to the fact that in reality not all field vectors point towards the observer we find an upper limit for this effect of $k^{2} \lesssim(19 \mathrm{~m})^{2}$. Since there is always a small uncertainty in determing the exact mass of a white dwarf, we also calculated an upper limit on $k^{2}$ assuming a lower mass of $1 M_{\odot}$. This leads to $k^{2} \lesssim(22 \mathrm{~m})^{2}$. An even more extreme assumption would be to use the maximum circular polarization predicted by all field strengths in the interval $1.4 \cdot 10^{4}-5.3 \cdot 10^{4}$ (reached at $5.3 \cdot 10^{4} \mathrm{~T}$ ) for the whole stellar disk. Then we obtain $V_{\lambda, \max } / I_{\lambda, \text { tot }}=48.3 \%$ and an upper limit of $k^{2} \lesssim(30.5 \mathrm{~m})^{2}$. Independent from any dipole model and without any reference to radiative transfer calculations the assumption of $100 \%$ emerging polarization leads to $k^{2} \lesssim(45 \mathrm{~m})^{2}$. In order to compare the quality of our method with previous similar results from Solanki et al. 8] we also set new upper limits on the NGT parameter $\ell_{\star}^{2}$ wich also causes gravitational birefringence in case of $\ell_{\star}^{2} \neq 0$. Assuming $V_{\lambda, \max } / I_{\lambda, \text { tot }}=26.5 \%$ leads to $\ell_{\star}^{2} \lesssim(1.8 \mathrm{~km})^{2}$ in contrast to $\ell_{\star}^{2} \lesssim(4.9 \mathrm{~km})^{2}$, determined for the white dwarf GRW $+70^{\circ} 8247$.

\section{CONCLUSIONS}

We have used spectropolarimetric observations of the massive $\left(1.35 M_{\odot}\right)$ white dwarf RE J0317-853, to impose new strong constraints on the birefringence of spacetime in the presence of a gravitational field. Such an effect is predicted by new physics in the form of a direct coupling of the electromagnetic field to auxiliary gravitational fields. Since the gravity-induced birefringence of space violates the Einstein equivalence principle, our analysis also provides a test of this foundation of general relativity and other metric theories of gravity. We consider as a specific case a metric-affine theory that couples the electromagnetic field with torsion and for which a static spherically symmetric field has been found [20]. The data provide an upper limit for the relevant coupling constant $k^{2}$ of $(19 \mathrm{~m})^{2}-$ or $(45 \mathrm{~m})^{2}$ for the most conservative assumptions on. Considerably tighter limits based on the same astronomical source could be provided by measurements of circular polarization in the FUV (in particular associated with Ly $\alpha$ absorption features) and also by a consistent model for the magnetic field geometry which reproduces the spectropolarimetry measurements in the optical.

We are grateful to Dayal T. Wickramasinghe and Friedrich $W$. Hehl for helpful remarks and valuable discussions. This research has made use of NASA's Astrophysics Data System Abstract Service.
[1] D. Colladay, V.A. Kostelecky, Phys. Rev. D 58, 116002 (1998) and references therein.

[2] V.A. Kostelecky, M. Mewes, Phys. Rev. Lett. 87, 251304 (2001) and Phys. Rev. D 66, 056005 (2002)

[3] C.D. Froggatt, H.B. Nielsen, Origin of Symmetries, World Scientific, Singapore, 1991

[4] C.M. Will, Theory and Experiment in Gravitation Physics, Second Edition, (Cambridge, 1993).

[5] W.-T. Ni, Phys. Rev. Lett. 38, 301 (1977).

[6] W.-T. Ni, in Precision Measurements and Fundamental Constants II, U.S. National Bureau of Standards Publication 617 (U.S. GPO, Washington D.C., 1984).

[7] S.K. Solanki, M.P. Haugan, Phys. Rev. D 53, 997 (1996)

[8] S.K. Solanki, M.P. Haugan, R.B. Mann, Phys. Rev. D 59, 047101 (1999).

[9] M.D. Gabriel et al., Phys. Rev. D. 43, 308 (1991).

[10] M.D. Gabriel et al., Phys. Rev. D. 43, 2465 (1991).

[11] M.P.Haugan, T.F. Kauffmann, Phys. Rev. D 52, 3168 (1995).

[12] J.W. Moffat, Phys. Letts. B 335, 447 (1995)

[13] T. Damour, S. Deser, J. McCarthy, Phys. Rev. D 45, R3289 (1992).

[14] M. Clayton, L. Demopoulos, J. Legare, GRG 30, 1501 (1998).

[15] F.W. Hehl et al., Phys. Repts. 258, 1 (1995).
[16] G.F. Rubilar, Y.N. Obukhov, F.W. Hehl, Class. Quantum Grav. 20 L185 (2003), Preprint gr-qc/0305049.

[17] Y. Itin, F.W. Hehl, Preprint gr-qc/0307063.

[18] R.A. Puntigam, C. Laemmerzahl, F.W. Hehl, Class. Quantum Grav. 14, 1347 (1997).

[19] R. Tresguerres, Z. Phys. C 65, 347 (1995).

[20] R. Tresguerres, Phys. Lett. A 200, 405 (1995).

[21] W.A. Shurcliff, Polarized Light. Harvard University Press, Harvard, Mass (1962).

[22] Magnetic Fields Across the Hertzsprung-Russell Diagram, ASP Conf. Proc. Vol. 248, p.479., 2001.

[23] J.D. Landstreet, Astron. Astrophys. Rev.4, 35 (1992)

[24] C.W. Allen, Astrophysical Quantities (Athlone, 1973)

[25] Stift M.J., Mon. Not. R. Astron. Soc. 172, 133-139 (1975)

[26] O. Preuss, PhD Thesis, Preprint gr-qc/0305083

[27] S.K. Solanki et al., Phys. Rev. D 69062001 (2004)

[28] S. Jordan, Astron. Astrophys.265, 570 (1992)

[29] M.A. Barstow, S. Jordan, D. O'Donoghue, M.R. Burleigh, R. Napiwotzki, M.K. Harrop-Allin, Mon. Not. R. Astron. Soc. 277, 971-985 (1995)

[30] M.R. Burleigh, S. Jordan, W. Schweizer, Astrophys. J. 510, L37-L40 (1999)

[31] S. Jordan, M.R. Burleigh, Proc. of the 11th Europ. Workshop on White Dwarfs, ASP Conf. Ser. 169, p. 235 (1999) 